Manuelle Medizin 2013 $\cdot 51: 373-373$

DOI 10.1007/s00337-013-1064-2

Online publiziert: 28 . September 2013

(c) Springer-Verlag Berlin Heidelberg 2013

\section{Psczolla}

Muskuloskeletales Zentrum der Loreley-Kliniken St. Goar-Oberwesel

\title{
Manuelle Medizin bei Kindern
}

Manuelle Medizin bei Kindern - dieses Thema ist seit mehreren Jahren virulent, nicht nur innerhalb unserer manualmedizinischen Community, sondern auch in der Diskussion mit unseren pädiatrischen und neuropädiatrischen Kollegen.

Zugegeben, auch unsere Protagonisten der Behandlung an Kindern und Säuglingen, die sich diesem Thema mit großem Ernst widmen, haben teilweise sehr unterschiedliche Vorstellungen, selbst Kontroversen untereinander gehabt und nach außen getragen.

Verschiedene Entitäten wurden in den letzten Jahren entwickelt, die teils theoretischen Charakter hatten, Schlagworte und Syndrome definiert, die nicht überall auf Gegenliebe stießen. Verfechtern solcher Begriffe standen entschiedene Gegner gegenüber, die Diskussion wurde nicht selten mit einer Schärfe ausgetragen, die dem Thema sicher nicht angemessen ist.

Nicht alles, was wir manualdiagnostisch und -therapeutisch in der manuellen Medizin anwenden, ist mit einem hohen Evidenzlevel belegt. Da unterscheiden wir uns nicht von vielen Fächern und Verfahren in der Medizin, ohne dass dies beispielsweise bei operativen Verfahren an Gelenken und Wirbelsäule groß diskutiert wird.

Nun ist es nicht so, dass in diesem Bereich nicht geforscht wird. Wir haben nicht wenige verdienstvolle Kolleginnen und Kollegen, die seit Jahren publizieren und dies mit hoher Qualität. Nur scheitern die Ansprüche an hohe Evidenzlevel bei Kindern und Säuglingen an besonderen ethischen Anforderungen. Dies gilt besonders für Kinder mit Behinderungen.

Wir hatten dieses Thema in der DGMM also schon lange im Visier, aber es wurde drängender, besprochen zu werden, da einige Kollegen in juristische Auseinandersetzungen verwickelt wurden, die ihre Behandlungsstrategien z. B. in ihrer Homepage dargestellt hatten.
So haben wir uns entschieden, eine Expertenrunde aufzufordern, Material zu sichten und zu einer Expertenmeinung zu kommen, die wir gemeinsam nach außen vertreten können. Im April dieses Jahres hat nun das DGMM-Präsidium Spezialisten dieses Behandlungsschwerpunkts nach Boppard eingeladen, und erfreulicherweise sind bis auf ganz wenige Kolleginnen und Kollegen, die aus nachvollziehbaren Gründen absagen mussten, alle gekommen. Es war eine von hohem Sachverstand und Engagement getragene Diskussion, die aufbauend auf der bekannten Literatur die verschiedenen Entitäten bestimmt hat. Die diagnostischen und therapeutischen Assessments wurden definiert.

So konnten wir den Wert der segmentalen Diagnostik als Vorbedingung zur spezifischen Therapie herausstellen.

Ferner haben wir Grundsätzliches zur Frage der rechtfertigenden Indikation für Röntgenaufnahmen bei Säuglingen und Kindern erarbeitet: Danach ergibt sich die Indikation zur Strahlendiagnostik an der HWS als Mittel der Diagnostik und nicht aufgrund des geplanten Einsatzes einer Therapieform. Das ist ein Paradigmenwechsel, selbst wenn diese Frage nicht von allen Experten übereinstimmend so gesehen wird. Dass einzelne therapeutische Techniken doch ein Röntgenbild erfordern sollen, bleibt ein spannender Diskussionspunkt, der uns weiter beschäftigen wird. Das Ergebnis kann sich sehen lassen. Mein Dank geht an alle Experten, Diskutanten und Moderatoren aus unserem Präsidium, Lothar Beyer, Hermann Locher und Wolfgang von Heymann, dem die redaktionelle Ausarbeitung zu verdanken ist. So soll das Ergebnis Ansporn sein, sich weiter mit dieser Frage zu beschäftigen.

Ein weiteres wichtiges Thema ist die Ergänzung unserer Jenenser Erklärung 2013 um das Thema osteopathische Verfahren für Physiotherapeuten. Wir konnten mit deren führenden Verbänden eine sehr gute Diskussion um die Zukunft der Anwendung osteopathischer Verfahren führen. Hier stimmt die gemeinsame Richtung, dass wir als DGMM diese Therapie als delegierbare Leistung, wie es sich bei der manuellen Therapie bewährt hat, fördern wollen und den Weg über den Heilpraktiker ablehnen. Weder uns Ärzten noch den Physiotherapeuten kann daran gelegen sein, nichtärztlichen oder -physiotherapeutischen Berufen zu ermöglichen, unseren gemeinsamen Patienten mit nicht qualitätsgesicherter Therapie parallel zu behandeln. Dafür, dass Politik und Krankenkassen auch unserer Sichtweise folgen, treten wir mit unserer berufspolitischen Zusammenarbeit ein.

Achten Sie auf unsere Patientenbroschüre über Osteopathie, die wir Ihren Patienten empfehlen. Diese Broschüre ist eine unterstützende Handreichung für Ihre tägliche Arbeit, die sie kostenlos direkt beim Verlag oder bei uns bestellen können.

Freuen Sie sich auf viele weitere Inhalte unseres neuen Hefts, das so manchen neuen Impuls für Sie bereithält.

\section{Herzlichst Ihr

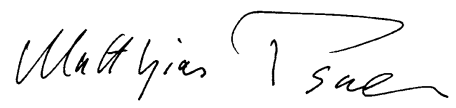

Dr. M. Psczolla

Präsident DGMM und Präsident DGMSM

\section{Korrespondenzadresse}

Dr. M. Psczolla
Muskuloskeletales Zentrum
der Loreley-Kliniken
St. Goar-Oberwesel
Hospitalgasse 11
55430 Oberwesel
dr.psczolla@loreley-kliniken.de

